\title{
Propuesta de sistema de costeo por órdenes para determinar los costos de producción en el Restaurant "Don Cuy", Huaraz - 2021
}

Proposal for a costing system by orders to determine production costs in the Restaurant "Don Cuy", Huaraz - 2021

Proposta de um sistema de custeio por encomendas para determinar os custos de produção no restaurante "Don Cuy", Huaraz - 2021

\author{
Diaz Huaman, Corina Yaniny \\ Consórcio da Universidade Cesar Vallejo, Chimbote, Peru \\ https://orcid.org/: 0000-0003-2209-7117 \\ E-mail: cydiazh@ucvvirtual.com
}

\author{
Dr. Vallejos Tafur, Juan Britman \\ Consórcio da Universidade Cesar Vallejo, Moyobamba, Peru \\ https://orcid.org/: 0000-0002-6328-806X \\ E-mail: jvallejost@ucvvirtual.com
}

\begin{abstract}
RESUMEN
Objetivo: Proponer un sistema de costeo por órdenes para determinar los costos de producción del Restaurant "Don Cuy", Huaraz - 2021. Metodología: La investigación se abordó desde el enfoque cuantitativo, tipo descriptivo, de diseño descriptivo-propositivo. La población y muestra estuvo constituida por todos los documentos de costos del primer trimestre del 2021 del restaurante. Los instrumentos aplicados fueron la guía de análisis documental, guía de cuestionario y guía de observación. Conclusión: no manejan un sistema de costos que permitan establecer cuál es el precio de venta, la ganancia y la utilidad; por lo que no se estiman como costos de producción; por lo que se diseñó de un sistema de costeo por órdenes que permitió tener valores reales de los platos, de la materia prima, mano de obra y de servicios implicados en los costos del restaurant; favoreciendo en la utilidad del negocio y la competitividad.
\end{abstract}

Palabras clave: Costos, Costeo por órdenes, Elementos del costo, Sistema de costeo, Análisis de costos.

\begin{abstract}
Objective: To propose an order costing system to determine the production costs of the "Don Cuy" Restaurant, Huaraz 2021. Methodology: The research was approached from the quantitative approach, descriptive type, descriptivepropositional design. The population and sample consisted of all the cost documents of the first quarter of 2021 of the restaurant. The instruments applied were the documentary analysis guide, the questionnaire guide and the observation guide. Conclusion: they do not manage a cost system to establish what the sale price, profit and utility are; therefore, they are not estimated as production costs; Therefore, a costing system was designed for orders that have real values of the dishes, raw materials, labor and services involved in the costs of the restaurant; favoring the utility of the business and competitiveness.
\end{abstract}

Keywords: Costs, Job order costing, Cost elements, Costing system, Cost analysis.

\section{RESUMO}

Objetivo: Propor um sistema de custeio por encomendas para determinar os custos de produção do Restaurante "Don Cuy", Huaraz - 2021. Metodologia: A pesquisa foi abordada a partir da abordagem quantitativa, tipo descritiva, desenho descritivo-proposicional. A população e amostra foi composta por todos os documentos de custos do primeiro trimestre de 2021 do restaurante. Os instrumentos aplicados foram o roteiro de análise documental, o roteiro do questionário e o roteiro de observação. Conclusão: não administram um sistema de custos que permita estabelecer qual é o preço de venda, o lucro e a utilidade; portanto, não são estimados como custos de produção; assim, foi desenhado um sistema de custeio por encomendas que permitisse ter valores reais dos pratos, matérias-primas, mão de obra e serviços envolvidos nos custos do restaurante; favorecendo a utilidade do negócio e a competitividade.

Palavras-chave: Custos, Custeio por ordem de serviço, Elementos de custo, Sistema de custeio, Análise de custos. 


\section{Introducción}

Toda empresa u organización dedicada a la venta de productos y servicios es imperativo que tenga una evaluación del sistema de costos, para poder incrementar su capacidad de mercado; asimismo, para ser competitiva frente a sus más cercanos competidores. No obstante, existen empresas que no ejecutan la evaluación adecuada de sus costos, que les facilite manejar elementos confiables sobre la información del negocio; de igual forma, que contribuya a la toma de decisiones administrativas.

Es así, como Nima (2018), precisa que: "Uno de los principales problemas que atraviesa este tipo de empresas es el escaso o nulo conocimiento sobre el manejo y control de sus costos lo que los lleva, muchas veces, a tener pérdidas y a desaparecer como empresa" (p.16).

Ante esta situación, se encontraron problemas a nivel internacional; tal como lo expresan Cárdenas y Rodríguez (2018), sobre el Restaurant del Sanatorio de Agua de Dios, ubicado en Colombia, expresa que: "la entidad maneja un sistema de costos que centra su operación en el cálculo de los costos por unidad funcional en la que se puede identificar un costeo general de los elementos indirectos y mano de obra" (p. 17).

Fonseca (2018), refiere acerca de los problemas que afrontó el Restaurant Paradero Don Gabriel están asociados a:

[...] fijar los precios de sus productos de forma empírica, lo cual conlleva a que la empresa desconozca con certeza cuánto debe costar la elaboración de sus platos de comidas, esto provoca que los precios pueden estar por debajo del costo real, provocándose pérdidas desapercibidas por el propietario. (p. 4)

A nivel nacional, se tiene respecto al Restaurant El Dorado ubicado en Chimbote, Urquiaga (2019), estableció que: "se debe conocer cómo es que se vienen asignando los costos de producción en el Restaurant El Dorado, así mismo conocer cuál es su margen de ganancia por cada plato que ofrece" (p.11).

Por su parte, Olivera (2019), respecto al Restaurant Turístico Tradiciones SAC. Chiclayo, enunció que como problema fundamental del estudio consideró los costos vinculados a la preparación de sus platillos; por lo que se hacía necesario saber cuáles eran sus costos; del mismo modo, establecer un valor para su venta que fuera el más adecuado, tanto para la empresa como para sus comensales; todo lo cual se vea reflejado en el beneficio del cliente. (p. 7)

Asimismo, Facundo (2018), sobre la problemática del restaurant turístico El Sabor de Mi Tierra, ubicado en San Ignacio en Cajamarca; estableció que este, en su momento no tenía un sistema de costeo apropiado; en ese sentido, el modo para estimarlos era de forma empírica; es por ello que no se tenía un valor fijo de lo correspondiente a los ingresos por plato de comida. (p. 17)

A nivel local, el Restaurant Don Cuy, ubicado en Huaraz en el departamento de Ancash; es un negocio dedicado al servicio de restaurant especializado en comidas típicas del Perú; en la actualidad de acuerdo con Ancash Noticias (2020), ha mantenido sus actividades según los protocolos establecidos debido a la pandemia del Covid 19, uniendo esfuerzos por mantener sirviendo a sus usuarios a través de un servicio de atención por aplicativo y utilizando delivery.

No obstante, su principal problema es que no llevan o poseen un sistema de costo apropiado, puesto que realizan un costeo empírico; en los que no realizan sus operaciones ajustados a los formatos o a un sistema en el que se visualice la trazabilidad de sus ingresos y gastos, lo que repercute al momento de hacer gestiones administrativas, asimismo, en su competitividad frente a otros negocios del mismo sector.

Es por ello, que se planteó como problema de investigación para el presente estudio la siguiente interrogante ¿De qué manera la propuesta de sistema de costeo por órdenes ayuda a determinar los costos de producción del restaurant "Don Cuy" Huaraz - 2021? 
Este estudio centró su justificación desde el punto de vista teórico, dado que servirá de base para otras investigaciones relacionadas con la variable evaluación de los costos, los métodos de costos y las definiciones que se derivan de este. Asimismo, la justificación metodológica para este estudio se fundamentó en que se emplearon los instrumentos de la guía de observación, la guía de cuestionario y la guía de análisis documental, lo que permitió que en el referido restaurant y otras organizaciones del mismo rubro en un futuro puedan realizar una evaluación de sus costos; al tiempo que los aportes teóricos que emergieron del mismo, se pueden constituir en insumos relevantes de carácter contable, administrativos y financieros; por lo tanto, apunta a coadyuvar a la optimización de sus procesos contables, financieros y toma de decisiones; específicamente del Restaurant Don Cuy Huaraz. Desde el punto de vista práctico, permitió a la organización, en este caso específico el Restaurant Don Cuy Huaraz, a resolver un problema que se vivía dentro de su organización; el mismo le impregnó de robustez todos sus procesos administrativos; incidiendo en la competitividad y su gestión operativa.

En ese sentido, el objetivo general de este estudio estuvo orientado a: Proponer un sistema de costeo por órdenes para determinar los costos de producción del Restaurant "Don Cuy", Huaraz - 2021.

Del mismo modo, como objetivos específicos se fijaron los siguientes: Describir los elementos del costo de producción en los que incurre, analizar los costos manejados por el Restaurant "Don Cuy", Huaraz - 2021 y diseñar una propuesta de costeo por órdenes de producción que le permita llevar un control de los principales platos de comida del Restaurant "Don Cuy", Huaraz - 2021.

Respecto a la hipótesis de este estudio, tal y como lo plantean Hernández, Fernández y Baptista (2014), algunas de las investigaciones cuantitativas no presentan hipótesis. Aquellas donde sí se formula hipótesis son las que presentan un alcance correlacional o explicativo, y en el caso de las descriptivas siempre y cuando se quiera pronosticar un hecho o una cifra.

Como antecedentes de la investigación a nivel internacional se tienen los trabajos de; Fonseca (2018), la investigación tuvo como objetivo evaluar los costos y su influencia en la fijación de precios de las comidas y bebidas en el Restaurant Paradero Don Gabriel, ubicado en Ecuador. La investigación es descriptiva-explicativa, con diseño no experimental. La muestra estuvo conformada por los trabajadores del restaurant. Como instrumentos de información se aplicó la entrevista estructurada y la guía de observación. Dentro de los resultados se tiene que: "el Restaurant Paradero Don Gabriel al contar con un diseño de herramienta de costos que ayude a la asignación de precios se vuelve más competitivo ante la competencia, ya que se basa en valores más reales" (Fonseca, 2018, p. 66).

Cárdenas y Rodríguez (2018), siendo el objetivo de la investigación diseñar un sistema de costeo basado en las actividades para el servicio de restaurante en el Sanatorio de Agua de Dios. La investigación es de tipo analítica y descriptiva, en la que se realizó un diagnóstico de la situación del costo del restaurant. La muestra estuvo constituida por 3 empleados del lugar y la información de sus registros. Como instrumentos se aplicó la guía de observación y la entrevista. Dentro de sus conclusiones se tiene que, se evidenciaron las debilidades predominantes en la distribución de los costos; por lo que de continuar el restaurant no alcanzaría la armonía en estos; asimismo, se estudiaron los insumos de abastecimientos para los primeros tres meses del año 2018 y contrastándolos con las ventas en el mismo lapso, encontrando una considerable perdida de dinero.

Perata, Freitas y Pesce (2016), el objetivo de la investigación es identificar y cuantificar los costos de cada uno de los productos vendidos y mejorar la manera en la toma de decisiones en cuanto al precio de venta de los productos. La investigación es de tipo investigación acción, con las cuatro fases: diagnóstico, investigación profunda, accion y evaluación. Como muestra se tomaron 4 personas trabajadoras de la empresa gastronómica. Los instrumentos de recolección de información aplicadas son las entrevistas, la ficha de observación, apoyados en la revisión documental. Como conclusiones se tiene que se desarrolló un método para la fijación de precios de ventas para una empresa multiproducto de gastronomía, fundamentado principalmente en los costos. Por lo que 
analizó la empresa para establecer una estructura de costos; en la que se propuso un modelo para mejorar en base a las necesidades detectadas en el diagnóstico.

Como antecedentes nacionales que dan soporte a la investigación, se tienen las investigaciones de Casanova (2020), la investigación tuvo como objetivo determinar en qué medida, la gestión de costos incide en la eficiencia en las ventas del servicio de restaurante en la empresa Chuck E Cheese's, ubicado en Trujillo. La investigación es de tipo aplicada, explicativa y mixta, de diseño de triangulación concurrente. La muestra estuvo constituida por 40 trabajadores de la referida empresa. Como instrumentos de recolección de información se aplicó un cuestionario de Likert. Como resultados se tiene que el $95 \%$ de los empleados opina que conocen poco o nada sobre la gestión de costo y de la forma de cómo se aplica en la empresa; mientras que el $75 \%$ de los trabajadores no tiene conocimiento acerca de los objetivos de ventas de la empresa.

Urquiaga (2019), cuya investigación tuvo como objetivo evaluar el costo de producción que contribuyera a determinar las ganancias del Restaurant El Dorado ubicado en Nuevo Chimbote, durante el año 2019. La misma es descriptiva, de diseño no experimental. La muestra estuvo constituida por el personal del referido restaurante y sus registros contables. Como instrumento se aplicaron la guía de entrevista y la guía de análisis documental. Dentro de sus conclusiones se tiene que: "el negocio al no tener conocimiento previo sobre un sistema de costos, desconoce la distribución de la mano de obra, solo realizan el pago al personal sin considerarlo dentro de sus costos" (Urquiaga, 2019, p. 32).

Olivera (2019), la investigación tuvo como objetivo realizar una propuesta de un sistema de costo ABC que contribuya a la determinación del costo del Restaurant Turístico Tradiciones SAC, ubicado en Chiclayo. La investigación es descriptiva, de diseño no experimental. La muestra estuvo conformada por 4 trabajadores de diversas áreas del restaurante. Como instrumento se aplicaron la guía de análisis documental y la guía de entrevista. Como conclusiones se tiene que la entidad ejecuta un sistema de costos tradicional; lo cual genera problemas en el manejo de costos; en ese sentido, no realiza una adecuada determinación de los costos.

Por otra parte, se aplicó el sistema de costo $A B C$, repercutiendo de forma significativa a la distribución que se realizan durante el procesamiento de costo del referido restaurante. 
Asimismo; Cartier y Yardin (1988) citado en Yardin (2002), establece sobre el costo que: "éste es un indicador que proporciona el apoyo necesario para obtener la dimensión o valor de algo; cuyo objetivo primordial es que sea de transacción en el comercio" (p.72).

Por su parte, Rincón (2005), precisó que; el costo en lo referido a la contabilidad representa un método para obtener información necesaria acerca del valor de bienes y servicios; que en este caso brinda alguna empresa; vinculados a las ganancias que proporcionan sus ventas; contribuyendo a la gestión operativa del negocio. (p. 269)

Para Rincón (2011), el costo: "se basa en las inversiones ejecutadas tomando en consideración las ganancias actuales y las que puedan generarse a lo largo del tiempo" (p. 10).

Samón, Cautín, Mustelier y Caraballo (2018), indicaron que: "el costo constituye una guía que sintetiza en términos económicos el efecto del uso de requerimientos monetarios, humanos y materiales en la ejecución de una tarea económica" (p. 77).

Sobre los objetivos del sistema de costos, Pérez (2003) y Reyes (s.f.) citado en Latorre (2016), indicaron que suministran los mecanismos esenciales para conservar el grado apropiado de materia prima, ayudando a obtener la asignación y regulación de compromisos, reduciendo cuestiones relacionados con materiales sobrantes, gastos indirectos, entre otros; asimismo, permite crear opciones para realizar variaciones en el proceso producto. (p. 515)

Adicionalmente, González (2017), sostiene sobre el objetivo del sistema de costo, son de gran importancia; puesto que desempeña un papel característico dentro de la administración del negocio; siendo empleado como instrumento primordial para ordenar, identificar y estudiar los costos. Asimismo, suministra los efectos que se tienen en el uso de los recursos; información acertada e importante que posibilita a la dirección tener un mejor enfoque para tomar decisiones. (p. 93)

De acuerdo con lo planteado en líneas anteriores, Hasan (2015), indicaron que: "el costeo del producto es uno de los objetos de la contabilidad de costo. El costeo apropiado de un producto es esencial para tomar las decisiones de gestión" (p. 1).

Concretando las ideas anteriores, Innocent y Chimezie (2013), expresaron que los costos constituyen el conjunto de medios sacrificados para obtener una meta determinada, que se traduce en la obtención de un bien o servicio. El eficaz desarrollo de un proyecto está vinculado con la regulación efectiva de los costos; en ese sentido, los costos se pueden verificar si previamente son estudiados y reconocidos. (p. 225)

Por su parte, Arellano et al. (2017), refiere que el costeo tiene como propósito "clasificar los costos y tasas de productividad de acuerdo con su empleo, de este modo desarrollar datos económicos de manera técnica y científica que contribuya alcanzar datos fiables para tomar decisiones" (p. 34).

Capa, García y Herrera (2019), argumentan que "los costos se constituyen como gastos monetarios originados en el desarrollo de construcción de un material o producto, sea por pagos, productos, mano de obra u otro" (p. 368).

Según Dal, Manfroi, Bortoli \& Theisen (2019) la importancia es, clasificar, analizar, acumular y asignar los costos correctos para una buena determinación de precios reales del producto (p. 19).

En ese sentido, Morillo y Cardozo (2016), expresaron que "la importancia del sistema de costeo radica en que, si se tienen estimaciones equivocadas de los costos, esto repercute en la toma de decisiones desacertadas dentro de la organización" (p. 92).

Falconí (2014), explicó que "la importancia de los sistemas de costeos implica lograr la utilidad real de la empresa, conocer los verdaderos precios de ventas que contribuya a competir con otros mercados, estableciendo estrategias de producción" (p. 63).

Según Reveles (2019), refirió que "los elementos de costos están constituidos por: materia prima, mano de obra y los costos de producción, también denominados costos indirectos" (párr. 15). 
Por su parte, para Rincón, Sánchez y Cardona (2019), sobre los elementos constitutivos del costo expresaron que: "estos representan la distribución básica de la clasificación del producto; dividiéndolos en costos fijos y variables o directos e indirectos" (p. 199)

De acuerdo Leite \& Renaud (2019) nos dice que los costos directos son aquellos costos que se encuentran relacionados a la elaboración de un bien o servicio, tales como materiales directos y mano de obra directa (p.3).

Reveles (2019), sobre los costos directos establecieron que: "son erogaciones que reúnen las tres cualidades (identificable, cuantificable y representativo), todo esto en la unidad de medida del satisfactor realizado" (párr. 5).

Para Cárdenas (2016), la materia prima directa es: "la empleada en el proceso de manufactura de un producto determinado; consiguiendo ser medida y atribuida a los costos del producto" (párr. 15).

Los costos de materia prima constituidos por materiales directos, Rojas (2007), expresa que: "componen la parte primordial de los costos de producción; siendo estos los materiales que se logran identificar de fácil manera en lo que se constituye el producto y que tiene un valor determinado" (p. 34).

Respecto a los costos de mano de obra, Artieda (2015), señalaron que: "estos son de gran importancia; puesto que, cumplen una función decisiva en los costos de mano de obra directa e indirecta; por lo cual la estimación de los tiempos de producción es relevante en la estimación de los costos directos" (p. 108).

Namazi (2016), expresó: "los costos de materiales directos y los costos de mano de obra directa se atribuyen a los objetos de costo seleccionados (por ejemplo, productos) directamente; por tanto, no buscan cualquier asignación de costos" (p.462).

En cuanto a los costos de producción o costos indirectos, Novoa, Gutiérrez y Bermúdez (2017), indicaron que: "estos constituyen la parte fundamental en la preparación del producto, en la que la materia prima y la mano de obra componen sus principales piezas" (p. 4).

Por su parte, Kato (2013), sobre los costos de producción precisaron que: “están relacionados con el progreso del proceso productivo; este suministra información sobre la cantidad de capital que representa la inversión de dinero" (p. 43).

Por lo expresado anteriormente, Gersil y Kayal (2016), establecieron que:

Los costos de los productos son un determinante significativo de las decisiones de las empresas tanto a corto como a largo plazo en términos de logro de objetivos. Al determinar el costo, se espera que las empresas consideren tanto las características del mercado (demanda) y el propio negocio. Los costos del producto se calculan por costo métodos de cálculo. (p. 79)

Morillo (2002), sobre los sistemas de costeo expresan que están conformados como pequeños sistemas de la contabilidad; los cuales manejan los datos referidos a los costos totales por concepto de fabricación del producto. Asimismo, refiere que entre estos se encuentran: sistemas de órdenes determinadas, por proceso, costeo predeterminados, costeos absorbentes, costeo variable, costeo basado en actividades (ABC), entre otros. Precisando que los sistemas de costeo ABC y absorbente son de los más utilizados. (p. 8)

Por su parte Macías, Reyes y Hurel (2020), establecen sobre el método de costeo absorbente que: "representa un mecanismo de costeo de inventarios en la que los valores asociados a la manufactura se asumen como costos inventariables" (p. 2).

Según Parra, Mondragón y Peña (2014), refieren que el costeo por absorción: "las empresas toman en cuenta los costos indirectos asociados a la manufactura o al servicio (variables y fijos) como el costo del bien o servicio" (p. 116)

Adicionalmente, Lima y Araújo (2017), respecto al costeo absorbente, establecen que: 
El método de absorción se implementa fácilmente, ya que todos los costos son absorbidos por el costo unitario. Esta apropiación de todos los costos (directos e indirectos, fijos y variables) ocasionados por el uso de los recursos productivos para los bienes elaborados, y solo los de producción, ocurre dentro del ciclo operativo interno. Todos los gastos relacionados con el esfuerzo de fabricación que se distribuyen a todos los productos elaborados. (p. 529)

Respecto a la asignación del costo según el costeo absorbente, el Ministerio de Economía y Finanzas (2016), estableció que: "se realiza fusionando los gastos directos con los gastos de otras tareas vinculadas con el proceso de producción. Siendo los elementos que componen el producto: la materia prima, mano de obra y los gastos indirectos y de manufactura" (párr. 3).

Por otro lado, Bunea (2013), sobre la asignación de costos bajo el método de absorción, expresó:

El costo de una unidad de producto bajo el método de costo por absorción consiste en materiales directos, mano de obra directa y gastos generales variables y fijos. El costeo de absorción asigna una parte de costo indirecto de fabricación para cada unidad de producto, junto con el costo variable de fabricación. (p. 124)

Por su parte, Almeida y Cunha (2017), refieren que:

El método de costeo $\mathrm{ABC}$, precisa que los recursos son asumidos por las actividades y estos son consumidos por los productos y servicios. La repartición de los recursos según las actividades y luego la asignación a los productos se ejecuta por medio de generación de costos. (p. 932)

La importancia del método ABC, según Dumitru y Ene (2018), expresan:

Es un método orientado a gestionar las actividades principales de la organización, contribuyendo al mejor establecimiento de los costos indirectos de una organización en cualquier contexto. Asimismo, ayuda a los métodos clásicos a conducir la contabilidad para acelerar los gastos en actividades. (p. 43-44)

Torre, Salete y Delgado (2017), expresan que "el costeo ABC puede ser aplicado a cualquier tipo de organización; sin embargo, son ampliamente usados por organizaciones que tienen diversos productos" (p. 647).

Adicionalmente, Bunea (2013), indicó sobre el costo de absorción: “el costo de producción incluye materiales, mano de obra directa y gastos de fabricación fijos y variables; los costos del período son de venta variable y fija y gastos administrativos" (p. 126).

Quiñonez (2020), refirió sobre el método de costeo ABC:

El sistema de costeo ABC estandariza cada una de las actividades del proceso productivo, mediante del consumo óptimo de recursos esto es tiempo, mano de obra, materiales; permita una aproximación real de asignación de erogaciones a cada parte del ciclo operacional. Es decir, no solo es un modelo para calcular, sino que agrega valor al sector donde se aplique. (p. 183)

Según Tran y Thao (2020), expresan sobre el método ABC:

Es un método que contribuye con las organizaciones a mejorar la eficacia en las operaciones y capacidad. De acuerdo con la información que suministra este método, los gerentes y encargados conocen las actividades les da valor agregado; asimismo, las actividades que pueden excluirse con la finalidad de ahorrar costos para la organización. Por otra parte, permite a los administradores establecer el costo de los productos con mayor exactitud, de este modo, los precios de los productos son más concisos. (p. 2625)

Por su parte, Alfarisi y Boediono (2021), refieren sobre los costos de producción "son los costos de los bienes comprados para ser procesados hasta su finalización, tanto antes como durante el periodo contable en curso; describen dos métodos de costos: costeo por órdenes y el método de costo por proceso" (p. 46) 
Greenberg y Schneider (2019), sustentan que el método de costeo por órdenes "inicia con la compra de materias primas y culmina con la venta del producto terminado" (p. 39).

Dewi, Musmini y Marvilianti (2019), argumentan sobre el método de costeo por órdenes:

La empresa puede estimar y conocer el costo total para una orden en todo momento, puesto que, los costos de producción están separados de modo que se puede calcular el costo de los bienes de producción de cada pedido de manera fácil. (p. 62)

Mulyanda y Evayani (2019), argumentan sobre el costeo por órdenes:

Es un método para determinar el costo de los bienes de producción en la que todos los gastos y costos de producción se añaden según una orden específica y costo de los bienes fabricados para cada producto se calcula estimando una división del costo total de producción para ese para ese pedido específico por el total de unidades del producto. (p. 428-429)

Idris (2019), refiere que el sistema de costo por orden:

Es usado ampliamente en empresas de servicios, como hospitales, oficinas de consultoría, estudios de derecho, estudios de cine, firmas de contabilidad, agencias de publicidad, talleres de reparación; utilizan un sistema de cobro de tarifas con costos de órdenes de trabajo para fines contables y de facturación. (p. 53)

Dewi, Tripaupi y Zukhri (2017), afirman que existen dos métodos que se emplean para la recaudación del costo de productos fabricados; "el primero es el método del precio de costo del producción o por orden y el segundo es el método de fijacion de precios o costo por proceso" (p. $518)$.

Por su parte, Putri y Desi (2019), argumentan que el método de costeo por órdenes:

Es muy importante en la toma de decisiones, iniciando con la orden de compra (PO) del cliente, la empresa debe calcular inmediatamente el costo de producción con sumo cuidado y precisión para estimar el precio de venta del producto. En este método los costos de producción se recopilan para una orden específica y el costo de producción por unidad producida se calcula dividiendo el costo total de producción del pedido por el número de unidades del producto en el pedido. (p. 349)

Hilmiyati, et. al. (2020), argumentan que "el sistema de cálculo de costos fundamentado en órdenes o pedidos se emplea para empresas que producen diversos productos durante un periodo determinado. Este método se emplea en trabajos de producción en base a pedidos de los clientes" (p. 562).

Afifah y Gunawan (2020), sostienen que "el costo de la tarjeta de pedidos u órdenes funciona como una cuenta de respaldo, que se emplea para recopilar y registrar en detalle los costos de producción de cada pedido de producto en cuestión" (p. 141).

Sitanggang, Sion y Suriyanti (2020), expresan que:

El cálculo del costo por órdenes de trabajo es una forma de cobrar el costo del producto para bienes o productos hechos por encargo. Cuando se recibe un pedido inmediatamente se emite una orden para fabricar el producto de acuerdo con las especificaciones de cada orden. (p. 169).

\section{PROCEDIMIENTOS METODOLOGICOS}

Según Palella y Martins (2012), las técnicas de recolección de información "constituyen los diversos modos de conseguir la información" (p. 115). Mientras que los instrumentos de recolección de información; Arias (2006), expresa que "son las estrategias que se utilizan para agrupar y distribuir la información" (p. 111).:

\section{A. Documental}

Sapienza: International Journal of Interdisciplinary Studies | Vol. 3 | n. 1 | Jan-Mar | 2022 | e-ISSN: 2675-9780 
De acuerdo con Balestrini (2006), el análisis documental "consiste en el análisis de fuentes documentales a través de la lectura para buscar información de interés en el texto escrito que sean relevantes para la investigación" (p. 152).

Nos permitió recoger información sobre la gestión administrativa y contable del Restaurant "Don Cuy".

\section{B. Encuesta}

De acuerdo a Rodríguez (2019), expresa que "se puede obtener datos a través de respuestas que los encuestados nos proporcionan, de acuerdo a las preguntas que son realizadas por el encuestador".

Por medio de esta guía que conto con 22 preguntas sobre costos, realizadas al dueño y gerente, nos ayudó a tener más información cierta sobre cómo se está manejando su sistema de costeo del restaurant "Don Cuy

\section{Observación}

Según Arias (2006), expresa que: "la observación es una técnica que se fundamenta en ver o captar a través de la vista, de manera ordenada, de los eventos que se generan en cualquier contexto de acuerdo con los fines de la investigación" (p. 69).

Con la que se recolectó la información necesaria a través del personal encargado del restaurante, del cual se obtuvieron los costos intervinientes para la preparación de los platos.

Al realizar estos procedimientos nos dan mayor información siendo necesarias para poder analizar en que circunstancias se encuentra el manejo de costeo del restaurant ya que al momento de entrevistar puedan llegar a omitir datos, por ende, se realiza el análisis documental para corroborar los datos que se puedan estar plasmados en sus archivos para el conocimiento de sus costos de cierto periodo.

Por lo tanto, los aspectos éticos fueron considerados para la discreción de los datos proporcionados por la empresa y la utilización de datos reales facilitados.

El restaurant "Don Cuy" ubicado en la Av. Centenario No 2621 urb. Palmira, Áncash - Huaraz Independencia, con RUC $N^{\circ} 20571440173$, es un negocio familiar dedicado al servicio de comidas típicas, dando inicio a sus actividades el 04 de diciembre del 2012 contando con un pequeño local, donde cada integrante de la familia tenía una función ya que ellos mismos elaboraban sus platos típicos para hacer crecer su empresa y a la vez sea reconocida como uno de las mejores marcas. Ya con el transcurrir del tiempo cuentan con una infraestructura moderna y propia manteniéndose en vigencia 8 años en el mercado, siendo uno de los mejores restaurants de la ciudad de Huaraz.

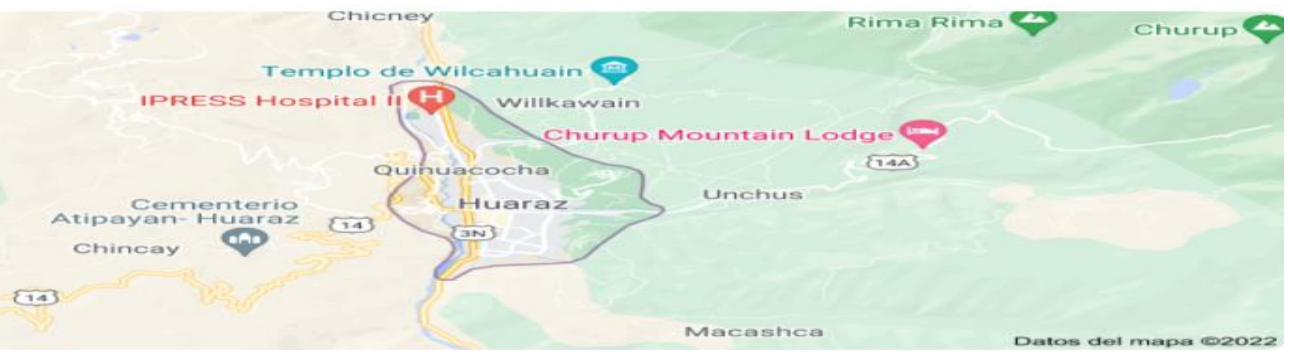

Huaraz ciudad peruana, capital del departamento de Áncash, y la segunda ciudad más poblada del departamento. Está situada a orillas del río Santa, se encuentra al centro norte del país y al norte de Lima. 


\section{RESULTADO Y DISCUCIÓN \\ DESCRIBIR LOS COSTOS}

El Restaurant "Don Cuy" cuenta con una variedad de platos a la carta donde asignación de sus costos de cada plato no están bien definidas. A continuación, se presentan los platos que se tomaron como muestra, de modo que, según la empresa los costos son considerados de la siguiente manera.

Costos de los insumos utilizados para la elaboración de los platos.

Costos de los insumos utilizados para la elaboración de los platos.

CUADRO N$^{\circ} 01$ Costos de los insumos para el Chancho al Palo

\begin{tabular}{|c|c|c|c|c|}
\hline Insumos & Cantidad & U.M & $\begin{array}{c}\text { Precio } \\
\text { U.M }\end{array}$ & $\begin{array}{c}\text { Cantidad } \\
\text { Total }\end{array}$ \\
\hline Chancho & 300 & $\mathrm{Gr}$ & 14 & 4.2 \\
\hline $\begin{array}{c}\text { Papas } \\
\text { blancas }\end{array}$ & 150 & Gr & 1 & 0.15 \\
\hline $\begin{array}{c}\text { Camote } \\
\text { sancochado }\end{array}$ & 90 & $\mathrm{Gr}$ & 3 & 0.27 \\
\hline $\begin{array}{c}\text { Papa } \\
\text { amarilla }\end{array}$ & 70 & Gr & 2.5 & 0.18 \\
\hline Choclo & 1 & Unidad & 0.8 & 0.8 \\
\hline Cebolla & 30 & Gr & 1.5 & 0.05 \\
\hline Lechuga & 2 & $\mathrm{Gr}$ & 1 & 0.01 \\
\hline Rocoto & 25 & $\mathrm{Gr}$ & 2.5 & 0.06 \\
\hline Limón & 1 & Unidad & 3 & 0.01 \\
\hline Hierva buena & 1 & Gr & 1.5 & 0.01 \\
\hline Ají limo & 4 & Gr & 3 & 0.01 \\
\hline Sal & 5 & Gr & 1.2 & 0.01 \\
\hline \multicolumn{5}{|c|}{ MATERIALES AUXILIARES } \\
\hline Mote serrano & 100 & Gr & 5 & 0.50 \\
\hline Arroz & 120 & Gr & 3.5 & 0.42 \\
\hline \multicolumn{4}{|c|}{ TOTAL } & 6.68 \\
\hline
\end{tabular}

\section{Análisis:}

Para la preparación del chancho al palo, se muestra en el cuadro, los insumos empleados y la cantidad requerida, identificando el costo principal de cada ingrediente, siendo el monto de S/. 6.68 por plato. 


\begin{tabular}{|c|c|c|c|c|c|}
\hline $\begin{array}{c}\text { Enero } \\
\text { Costo Diario (39) }\end{array}$ & $\begin{array}{c}\text { Enero } \\
\text { Costo Mensual }\end{array}$ & $\begin{array}{c}\text { Febrero } \\
\text { Costo Diario (30) }\end{array}$ & $\begin{array}{c}\text { Febrero } \\
\text { Costo Mensual }\end{array}$ & $\begin{array}{c}\text { Marzo } \\
\text { Costo Diario (35) }\end{array}$ & $\begin{array}{c}\text { Marzo } \\
\text { Costo Mensual }\end{array}$ \\
\hline 163.80 & 4914.00 & 126.00 & 3654.00 & 147.00 & 4410.00 \\
\hline 5.85 & 175.50 & 4.50 & 130.50 & 5.25 & 157.50 \\
\hline 10.53 & 315.90 & 8.10 & 234.90 & 9.45 & 283.50 \\
\hline 7.02 & 210.60 & 5.40 & 156.60 & 6.30 & 189.00 \\
\hline 31.20 & 936.00 & 24.00 & 696.00 & 28.00 & 840.00 \\
\hline 1.95 & 58.50 & 1.50 & 43.50 & 1.75 & 52.50 \\
\hline 0.39 & 11.70 & 0.30 & 8.70 & 0.35 & 10.50 \\
\hline 2.34 & 70.20 & 1.80 & 52.20 & 2.10 & 63.00 \\
\hline 0.39 & 11.70 & 0.30 & 8.70 & 0.35 & 10.50 \\
\hline 0.39 & 11.70 & 0.30 & 8.70 & 0.35 & 10.50 \\
\hline 0.39 & 11.70 & 0.30 & 8.70 & 0.35 & 10.50 \\
\hline \multirow[t]{2}{*}{0.39} & 11.70 & 0.30 & 8.70 & 0.35 & 10.50 \\
\hline & & 0.00 & & 0.00 & \\
\hline 19.50 & 585.00 & 15.00 & 435.00 & 17.50 & 525.00 \\
\hline 16.38 & 491.40 & 12.60 & 365.40 & 14.70 & 441.00 \\
\hline 260.52 & 7815.60 & 200.40 & 5811.60 & 233.80 & 7014.00 \\
\hline
\end{tabular}

\section{Análisis:}

En este cuadro se refleja el costo del chancho al palo de cada mes variando en la cantidad consumida por el primer trimestre del año. (marzo).

\section{CUADRO $\mathrm{N}^{\circ} 02$ Costos de los insumos para el Chancho al Cilindro}

\begin{tabular}{|c|c|c|c|c|}
\hline Insumos & Cantidad & U.M & $\begin{array}{c}\text { Precio } \\
\text { U.M }\end{array}$ & Cantidad Total \\
\hline Chancho & 300 & $\mathrm{Gr}$ & 16 & 4.80 \\
\hline Mote serrano & 100 & $\mathrm{Gr}$ & 5 & 0.50 \\
\hline Camote sancochado & 90 & $\mathrm{Gr}$ & 3 & 0.27 \\
\hline Papa amarilla & 70 & $\mathrm{Gr}$ & 2.5 & 0.18 \\
\hline Choclo & 1 & $\mathrm{Unidad}$ & 0.8 & 0.80 \\
\hline Cebolla & 30 & $\mathrm{Gr}$ & 1.5 & 0.05 \\
\hline Lechuga & 2 & $\mathrm{Gr}$ & 1 & 0.01 \\
\hline Rocoto & 25 & $\mathrm{Gr}$ & 2.5 & 0.06 \\
\hline Limón & 1 & $\mathrm{Unidad}$ & 3 & 0.01 \\
\hline Hierva buena & 1 & $\mathrm{Gr}$ & 1.5 & 0.01 \\
\hline Ajíl limo & 4 & $\mathrm{Gr}$ & 3 & 0.01 \\
\hline Sal & 5 & $\mathrm{Gr}$ & 1.2 & 0.01 \\
\hline Ají amarillo & 6 & $\mathrm{Gr}$ & 3.5 & 0.02 \\
\hline Ajos & 5 & $\mathrm{Gr}$ & 11 & 0.06 \\
\hline \multicolumn{2}{|c|}{ MATERIALES AUXILIARES } & $\mathrm{Gr}$ & 1 & 0.15 \\
\hline Papas blancas & 150 & $\mathrm{Gr}$ & 3.5 & 0.42 \\
\hline Arroz & 120 & & & 7.36 \\
\hline \multicolumn{2}{|c|}{ TOTAL } & & & \\
\hline
\end{tabular}

Análisis: 
Para la preparación del chancho al cilindro, se muestra en el cuadro, los insumos empleados y la cantidad requerida, identificando el costo principal de cada ingrediente, siendo el monto de S/. 7.36. por plato.

\begin{tabular}{|r|r|r|r|r|r|}
\hline $\begin{array}{r}\text { Enero } \\
\text { Costo Diario (39) }\end{array}$ & $\begin{array}{c}\text { Enero } \\
\text { Costo Mensual }\end{array}$ & $\begin{array}{c}\text { Febrero } \\
\text { Costo Diario (32) }\end{array}$ & $\begin{array}{c}\text { Febrero } \\
\text { Costo Mensual }\end{array}$ & $\begin{array}{c}\text { Marzo } \\
\text { Costo Diario (32) }\end{array}$ & $\begin{array}{c}\text { Marzo } \\
\text { Costo Mensual }\end{array}$ \\
\hline 187.20 & 5616.00 & 153.60 & 4454.40 & 153.60 & 4608.00 \\
\hline 19.50 & 585.00 & 16.00 & 464.00 & 16.00 & 480.00 \\
\hline 10.53 & 315.90 & 8.64 & 250.56 & 8.64 & 259.20 \\
\hline 7.02 & 210.60 & 5.76 & 167.04 & 5.76 & 172.80 \\
\hline 31.20 & 936.00 & 25.60 & 742.40 & 25.60 & 768.00 \\
\hline 1.95 & 58.50 & 1.60 & 46.40 & 1.60 & 48.00 \\
\hline 0.39 & 11.70 & 0.32 & 9.28 & 0.32 & 9.60 \\
\hline 2.34 & 70.20 & 1.92 & 55.68 & 1.92 & 57.60 \\
\hline 0.39 & 11.70 & 0.32 & 9.28 & 0.32 & 9.60 \\
\hline 0.39 & 11.70 & 0.32 & 9.28 & 0.32 & 9.60 \\
\hline 0.39 & 11.70 & 0.32 & 9.28 & 0.32 & 9.60 \\
\hline 0.39 & 11.70 & 0.32 & 9.28 & 0.32 & 9.60 \\
\hline & & & & & \\
\hline 0.78 & 23.40 & 0.64 & 18.56 & 0.64 & 19.20 \\
\hline 2.34 & 70.20 & 1.92 & 55.68 & 1.92 & 57.60 \\
\hline & & & & 0.00 & \\
\hline 5.85 & 175.50 & 4.80 & 139.20 & 4.80 & 144.00 \\
\hline 16.38 & 491.40 & 13.44 & 389.76 & 13.44 & 403.20 \\
\hline 287.04 & 8611.20 & 235.52 & 6830.08 & 235.52 & 7065.60 \\
\hline & & & &
\end{tabular}

\section{Análisis:}

En este cuadro se refleja el costo del chancho al cilindro de cada mes variando en la cantidad consumida por el primer trimestre del año. (marzo)

\section{CUADRO Nº3 Costos de los insumos para el Don Cuisano}

\begin{tabular}{|c|c|c|c|r|}
\hline Insumos & Cantidad & U.M & $\begin{array}{c}\text { Precio } \\
\text { U.M }\end{array}$ & $\begin{array}{c}\text { Cantidad } \\
\text { Total }\end{array}$ \\
\hline Cuy & $1 / 2$ & $\mathrm{Gr}$ & 22 & 11 \\
\hline $\begin{array}{c}\text { Papas } \\
\text { blancas }\end{array}$ & 150 & $\mathrm{Gr}$ & 1 & 0.15 \\
\hline Arroz & 70 & $\mathrm{Gr}$ & 3.5 & 0.25 \\
\hline $\begin{array}{c}\text { Mote de } \\
\text { trigo }\end{array}$ & 50 & $\mathrm{Gr}$ & 4 & 0.2 \\
\hline Ají amarillo & 40 & $\mathrm{Gr}$ & 3 & 0.12 \\
\hline Ají colorado & 40 & $\mathrm{Gr}$ & 5 & 0.2 \\
\hline Ají mirasol & 40 & $\mathrm{Gr}$ & 5 & 0.2 \\
\hline Cebolla & 20 & $\mathrm{Gr}$ & 1 & 0.02 \\
\hline Lechuga & 2 & $\mathrm{Gr}$ & 1 & 0.01 \\
\hline Ajos & 3 & $\mathrm{Gr}$ & 11 & 0.03 \\
\hline Limón & 1 & $\mathrm{Unidad}$ & 3 & 0.01 \\
\hline Ají limo & 4 & $\mathrm{Gr}$ & 3 & 0.01 \\
\hline $\begin{array}{c}\text { Hierva } \\
\text { buena }\end{array}$ & 1 & $\mathrm{Gr}$ & 1.5 & 0.01 \\
\hline Sal & 6 & $\mathrm{Gr}$ & 1.2 & 0.01 \\
\hline Aceite & 1 & $\mathrm{Litro}$ & 8 & 2.00 \\
\hline \multicolumn{2}{|c|}{$\mathrm{TOTAL}$} & & 14.22 \\
\hline
\end{tabular}




\section{Análisis:}

Para la preparación del Don Cuisano, se muestra en el cuadro, los insumos empleados y la cantidad requerida, identificando el costo principal de cada ingrediente, siendo el monto de S/. 14.22 por plato.

\begin{tabular}{|c|c|c|c|c|c|}
\hline $\begin{array}{c}\text { Enero } \\
\text { Costo Diario (60) }\end{array}$ & $\begin{array}{c}\text { Enero } \\
\text { Costo Mensual }\end{array}$ & $\begin{array}{c}\text { Febrero } \\
\text { Costo Diario (52) }\end{array}$ & $\begin{array}{c}\text { Febrero } \\
\text { Costo Mensual }\end{array}$ & $\begin{array}{c}\text { Marzo } \\
\text { Costo Diario (42) } \\
\end{array}$ & $\begin{array}{c}\text { Marzo } \\
\text { Costo Mensual }\end{array}$ \\
\hline 660.00 & 19800.00 & 572.00 & 16588 & 462.00 & 13860.00 \\
\hline 9.00 & 270.00 & 7.80 & 226.2 & 6.30 & 189.00 \\
\hline 15.00 & 450.00 & 13.00 & 377 & 10.50 & 315.00 \\
\hline 12.00 & 360.00 & 10.40 & 301.6 & 8.40 & 252.00 \\
\hline 7.20 & 216.00 & 6.24 & 180.96 & 5.04 & 151.20 \\
\hline 12.00 & 360.00 & 10.40 & 301.6 & 8.40 & 252.00 \\
\hline 12.00 & 360.00 & 10.40 & 301.6 & 8.40 & 252.00 \\
\hline 1.20 & 36.00 & 1.04 & 30.16 & 0.84 & 25.20 \\
\hline 0.60 & 18.00 & 0.52 & 15.08 & 0.42 & 12.60 \\
\hline 1.80 & 54.00 & 1.56 & 45.24 & 1.26 & 37.80 \\
\hline 0.60 & 18.00 & 0.52 & 15.08 & 0.42 & 12.60 \\
\hline 0.60 & 18.00 & 0.52 & 15.08 & 0.42 & 12.60 \\
\hline 0.60 & 18.00 & 0.52 & 15.08 & 0.42 & 12.60 \\
\hline 0.60 & 18.00 & 0.52 & 15.08 & 0.42 & 12.60 \\
\hline 120.00 & 3600.00 & 104.00 & 3016 & 84.00 & 2520.00 \\
\hline 853.20 & 25596.00 & 739.44 & 21443.76 & 597.24 & 17917.20 \\
\hline
\end{tabular}

\section{Análisis:}

En este cuadro se refleja el costo del Don Cuisano de cada mes variando en la cantidad consumida por el primer trimestre del año. (marzo) 
Costos de la Mano de Obra y otros GRATIFICACIÓN

CUADRO N04 Remuneración mensual a los trabajadores

\begin{tabular}{|c|c|c|c|c|c|c|c|}
\hline Cargo & $\begin{array}{l}\text { Días laborales } \\
\text { en el mes }\end{array}$ & $\begin{array}{l}\text { Cantidad de } \\
\text { personal }\end{array}$ & $\begin{array}{c}\text { Remuneración } \\
\text { mensual }\end{array}$ & & Total & $\begin{array}{r}\text { C } \\
\text { TRII } \\
\end{array}$ & $\begin{array}{l}\text { OSTO } \\
\text { VESTRA } \\
\text { L }\end{array}$ \\
\hline Cocinero & 30 & 1 & $1,200.00$ & $\mathrm{~S} /$ & $1,200.00$ & $\mathrm{~S} /$ & $3,600.00$ \\
\hline Cocinero & 30 & 1 & $1,200.00$ & $\mathrm{~S} /$ & $1,200.00$ & $\mathrm{~S} /$ & $3,600.00$ \\
\hline Cocinero & 30 & 1 & $1,400.00$ & $\mathrm{~S} /$ & $1,400.00$ & $\mathrm{~S} /$ & $4,200.00$ \\
\hline Ayudante de cocina & 30 & 3 & 930.00 & $\mathrm{~S} /$ & $2,790.00$ & $\mathrm{~S} /$ & $8,370.00$ \\
\hline Cajero & 30 & 1 & $1,200.00$ & $\mathrm{~S} /$ & $1,200.00$ & $\mathrm{~S} /$ & $3,600.00$ \\
\hline Barra & 30 & 1 & $1,000.00$ & $\mathrm{~S} /$ & $1,000.00$ & $\mathrm{~S} /$ & $3,000.00$ \\
\hline Mesero & 30 & 8 & 930.00 & $\mathrm{~S} /$ & $7,440.00$ & $\mathrm{~S} /$ & $22,320.00$ \\
\hline Personal de limpieza & 30 & 1 & 930.00 & $\mathrm{~S} /$ & 930.00 & $\mathrm{~S} /$ & $2,790.00$ \\
\hline Almacenero & 30 & 1 & 930.00 & $\mathrm{~S} /$ & 930.00 & $\mathrm{~S} /$ & $2,790.00$ \\
\hline Gerente & 30 & 1 & $2,000.00$ & $\mathrm{~S} /$ & $2,000.00$ & $\mathrm{~S} /$ & $6,000.00$ \\
\hline Administrador & 30 & 1 & $1,500.00$ & $\mathrm{~S} /$ & $1,500.00$ & $\mathrm{~S} /$ & $4,500.00$ \\
\hline \multicolumn{4}{|c|}{ TOTAL } & $\mathbf{S} /$ & $21,590.00$ & $\mathbf{S} /$ & $64,770.00$ \\
\hline
\end{tabular}

\section{Análisis:}

En el cuadro de planilla se puede observar la remuneración del mes de marzo, realizando un desembolso de S/. 21,590.00, cabe resaltar que solo la mitad de sus trabajadores se encuentra en planilla.

\begin{tabular}{|c|c|c|r|r|}
\hline № & ORDEN DE PEDIDO & $\%$ & \multicolumn{1}{c|}{ MOI } & \multicolumn{1}{c|}{ MOD } \\
\hline 1 & Chancho al Palo & $12 \%$ & 11.53 & 11.92 \\
\hline 2 & Chancho al Cilindro & $11 \%$ & 10.57 & 10.92 \\
\hline 3 & Don Cuisano & $14 \%$ & 13.45 & 13.90 \\
\hline
\end{tabular}

\section{Análisis:}

En este cuadro se muestra el costo de la mano de Obra Indirecta y Directa de los platos más vendidos por el restaurant "Don Cuy", por lo que tomo en cuenta el salario de los cocineros y ayudantes ya que ellos son los que elaboran de manera directa los potajes. Para el cálculo se tomó en cuenta una tarjeta de tiempo de cada trabajador, teniendo en cuenta las horas trabajadas para la elaboración de cada plato.

El porcentaje cada potaje se obtuvo de la cantidad de platos vendidos por día un promedio de 350 vendidos, teniendo encueta que son 22 platos que ofrecen restaurant.

\section{CUADRO N$^{\circ} 05$ Pagos mensuales del suministro}




\begin{tabular}{|l|r|lr|}
\hline \multicolumn{1}{|c|}{ Suministros } & \multicolumn{1}{c|}{ Total } & \multicolumn{2}{c|}{$\begin{array}{c}\text { COSTO } \\
\text { TRIMESTRAL }\end{array}$} \\
\hline Descartables & $1,500.00$ & $\mathrm{~S} /$ & $4,500.00$ \\
\hline Taxi & 400.00 & $\mathrm{~S} /$ & $1,200.00$ \\
\hline $\begin{array}{l}\text { Implementos de } \\
\text { oficina }\end{array}$ & 200.00 & $\mathrm{~S} /$ & 600.00 \\
\hline Marketing & 300.00 & $\mathrm{~S} /$ & 900.00 \\
\hline $\begin{array}{l}\text { Suministro de } \\
\text { limpieza }\end{array}$ & 700.00 & $\mathrm{~S} /$ & $2,100.00$ \\
\hline Leña & $1,600.00$ & $\mathrm{~S} /$ & $4,800.00$ \\
\hline Carbón & $1,200.00$ & $\mathrm{~S} /$ & $3,600.00$ \\
\hline \multicolumn{1}{|c|}{ Total } & $\mathbf{5 , 9 0 0 . 0 0}$ & $\mathrm{S} /$ & $\mathbf{1 7 , 7 0 0 . 0 0}$ \\
\hline \multicolumn{2}{|r|}{} \\
\hline
\end{tabular}

Análisis:

En el cuadro se puede observar que la empresa utilizo su suministro en el mes de marzo por un importe de $\mathrm{S} / 5,900.00$

CUADRO Nº6 Servicios básicos del mes de abril

\begin{tabular}{|l|rr|rr|}
\hline \multicolumn{1}{|c|}{ Servicios } & \multicolumn{2}{c|}{ Total } & \multicolumn{2}{c|}{$\begin{array}{c}\text { COSTO } \\
\text { TRIMESTRAL }\end{array}$} \\
\hline Luz & $\mathrm{S} /$ & $3,500.00$ & $\mathrm{~S} /$ & $10,500.00$ \\
\hline Agua & $\mathrm{S} /$ & $3,000.00$ & $\mathrm{~S} /$ & $9,000.00$ \\
\hline Gas & $\mathrm{S} /$ & $2,700.00$ & $\mathrm{~S} /$ & $8,100.00$ \\
\hline Internet, teléfono & $\mathrm{S} /$ & 300.00 & $\mathrm{~S} /$ & 900.00 \\
\hline \multicolumn{1}{|c|}{ Total } & $\mathrm{S} /$ & $\mathbf{9 , 5 0 0 . 0 0}$ & $\mathrm{S} /$ & $\mathbf{2 8 , 5 0 0 . 0 0}$ \\
\hline
\end{tabular}

\section{Análisis:}

En el cuadro se puede observar que la empresa utilizó sus servicios básicos en el mes de marzo por un importe de $S / 6,620.00$. 
DETALLE DE LOS CIF EN CADA ORDEN DE PRODUCCIÓN

\begin{tabular}{|l|r|r|r|r|}
\hline $\begin{array}{c}\text { Costos Indirectos de } \\
\text { Fabricación }\end{array}$ & $\begin{array}{c}\text { Monto Diario } \\
\text { de los CIF }\end{array}$ & $\begin{array}{c}\text { Chancho al } \\
\text { Palo 12\% }\end{array}$ & $\begin{array}{c}\text { Don } \\
\text { Chancho al } \\
\text { Cilindro 11\% }\end{array}$ & $\begin{array}{c}\text { Duisano } \\
\mathbf{1 4 \%}\end{array}$ \\
\hline Luz & 116.67 & 14.00 & 12.83 & 16.33 \\
\hline Agua & 100.00 & 12.00 & 11.00 & 14.00 \\
\hline Gas & 90.00 & 10.80 & 9.90 & 12.60 \\
\hline Internet, teléfono & 10.00 & 1.20 & 1.10 & 1.40 \\
\hline Descartables & 50.00 & 6.00 & 5.50 & 7.00 \\
\hline Suministro de limpieza & 35.00 & 4.20 & 3.85 & 4.90 \\
\hline Implementos de oficina & 6.67 & 0.80 & 0.73 & 0.93 \\
\hline Taxi & 13.33 & 1.60 & 1.47 & 1.87 \\
\hline Marketing & 10.00 & 1.20 & 1.10 & 1.40 \\
\hline Leña & 53.33 & 6.40 & 5.87 & 7.47 \\
\hline Carbón & 40.00 & 4.80 & 4.40 & 5.60 \\
\hline \multicolumn{1}{r|}{ TOTAL } & $\mathbf{1 3 8 0 . 0 4}$ & $\mathbf{6 3 . 0 0}$ & $\mathbf{5 7 . 7 5}$ & $\mathbf{7 3 . 5 0}$ \\
\hline
\end{tabular}

\section{Análisis:}

Se muestra los costos indirectos por mes que tiene en cuenta el restaurant para la elaboración de los platos más vendidos por día. Donde el Chancho al palo tiene un costo S/. $63+11.53$, chancho al Cilindro S/. 57.50+11.57 y el don Cuisano de S/. 73.50+13.55.

\section{HOJA DE COSTOS}

\begin{tabular}{|l|r|r|r|}
\hline \multicolumn{1}{|c|}{ CIERRE DE LA HOJA DE COSTOS } & \multicolumn{1}{c|}{$\begin{array}{c}\text { Chancho al } \\
\text { Palo }\end{array}$} & \multicolumn{1}{c|}{$\begin{array}{c}\text { Chancho al } \\
\text { Cilindro }\end{array}$} & \multicolumn{1}{c|}{$\begin{array}{c}\text { Don } \\
\text { Cuisano }\end{array}$} \\
\hline (+) MATERIALES DIRECTOS & 233.80 & 235.52 & 597.24 \\
\hline (+) MANO DE OBRA DIRECTA & 11.92 & 10.92 & 13.90 \\
\hline $\begin{array}{l}\text { (+) COSTOS INDIRECTO DE } \\
\text { FABRICACIÓN }\end{array}$ & 74.53 & 68.32 & 86.95 \\
\hline (=) TOTAL DE COSTO DE PRODUCCIÓN & $\mathbf{3 2 0 . 2 5}$ & $\mathbf{3 1 4 . 7 6}$ & $\mathbf{6 9 8 . 0 9}$ \\
\hline UNIDADES PRODUCIDAD & 35 & 32 & 42 \\
\hline COSTO UNITARIO & $\mathbf{9 . 1 5}$ & $\mathbf{9 . 8 4}$ & $\mathbf{1 6 . 6 2}$ \\
\hline
\end{tabular}

En el cuadro se muestra que para la hoja de costos se tomara en cuenta el material, mano de Obra y los CIF para cada elaboración de plato por día. 
ANALISIS DE LOS COSTOS

El cuadro se muestra, la suma de los tres elementos del costo, considerando el costo de producción por cada potaje, de modo que se refleja el costo unitario según costeo empírico del restaurant y costo según propuesta.

\begin{tabular}{|c|c|c|c|c|c|c|}
\hline & C.RESTAURANT & PROPUESTA & C.RESTAURANT & PROPUESTA & C.RESTAURANT & PROPUESTA \\
\hline $\begin{array}{l}\text { CIERRE DE LA HOJA DE } \\
\text { COSTOS }\end{array}$ & \multicolumn{2}{|c|}{ Chancho al Palo } & \multicolumn{2}{|c|}{ Chancho al Cilindro } & \multicolumn{2}{|c|}{ Don Cuisano } \\
\hline (+) MATERIALES DIRECTOS & 233.80 & 236.14 & 235.52 & 237.63 & 597.24 & 599.98 \\
\hline (+) MANO DE OBRA DIRECTA & 11.92 & 17.56 & 10.92 & 16.10 & 13.90 & 20.49 \\
\hline $\begin{array}{l}\text { (+) COSTOS INDIRECTO DE } \\
\text { FABRICACIÓN }\end{array}$ & 74.53 & 179.08 & 68.32 & 164.16 & 86.95 & 208.92 \\
\hline $\begin{array}{l}\text { (=) TOTAL DE COSTO DE } \\
\text { PRODUCCIÓN }\end{array}$ & 320.25 & 432.78 & 314.76 & 417.89 & 698.09 & 829.40 \\
\hline UNIDADES PRODUCIDAD & 35 & 35 & 32 & 32 & 42 & 42 \\
\hline COSTO UNITARIO & $\mathrm{S} / 9$ & $\mathrm{~S} / \mathrm{12}$ & $\mathrm{S} / 10$ & S/ 13 & S/ 17 & $\mathrm{~S} / \mathbf{2 0}$ \\
\hline
\end{tabular}

\section{Propuesta}

\begin{tabular}{|l|r|r|r|}
\hline Chancho al Palo & 25.00 & 12.00 & 13.00 \\
\hline Chancho al Cilindro & 25.00 & 13.00 & 12.00 \\
\hline Don Cuisano & 26.00 & 20.00 & 6.00 \\
\hline
\end{tabular}

Según el cuadro se puede observar la gran diferencia de precio.

Por lo tanto, se puede diferenciar de los tres platos que el más rentable es el chancho al palo, ya que genera mayor ganancia a la empresa y a la vez genera menor costo para su elaboración.

\section{DISCUSIÓN}

A través de los hallazgos obtenidos para el objetivo general de este estudio, el cual estuvo orientado a proponer un sistema de costeo por órdenes para determinar los costos de producción del Restaurant "Don Cuy", Huaraz - 2021, donde por medio de la guía de observación, la guía de análisis documental y el cuestionario realizados a dos de sus colaboradores, se precisó que en el Restaurant "Don Cuy" se presentan ciertas debilidades.

Cárdenas y Rodríguez (2018), indican que encontraron debilidades en la distribución de los costos; por lo que de continuar el restaurant no alcanzaría la armonía en estos; asimismo, se estudiaron los insumos de abastecimientos para los primeros tres meses del año 2018 y contrastándolos con las ventas en el mismo lapso, encontrando una considerable pérdida de dinero.

De acuerdo con esto, en el Restaurant Don Cuy se encontró que no manejan un sistema de costos en el que se precise los costos de los insumos, materias primas, costos de servicios básicos y generales, pago a trabajadores; en consecuencia, no se tienen estimados los costos de producción. 
Se considera de gran relevancia el haber diagnosticado problemas similares a los mostrados por Cárdenas y Rodríguez (2018) en su estudio, puesto que, previene al Restaurant Don Cuy a que sufra pérdidas económicas considerados en los costos de producción.

Casanova (2020), argumenta que dentro de sus resultados obtuvo que el $95 \%$ de los empleados opina que conocen poco o nada sobre la gestión de costo y de la forma de cómo se aplica en la empresa; mientras que el $75 \%$ de los trabajadores no tiene conocimiento acerca de los objetivos de ventas de la empresa.

En ese orden de ideas, de acuerdo a las entrevistas aplicadas a dos de sus colaboradores los trabajadores tienen escasos conocimientos acerca de cómo se gestionan de los costos, del control de inventario y productos, la realización de las compras, entre otros elementos que inciden directamente en llevar un buen control de los costos y que se visualicen las ganancias, utilidades, pérdidas.

Por su parte, es fundamental que los colaboradores de una organización tengan conocimientos acerca de la gestión en la que se desempeñan por lo menos los dueños y el gerente deben tener conocimientos mínimos sobre el costo de sus potajes a elaborar, para estimar si éste es rentable y genera utilidad.

Urquiaga (2019), quien en su estudio en el Restaurant El Dorado de Nuevo Chimbote, encontró que: "el negocio al no tener conocimiento previo sobre un sistema de costos, desconoce la distribución de la mano de obra, solo realizan el pago al personal sin considerarlo dentro de sus costos" (Urquiaga, 2019, p. 32).

De manera similar a lo que argumenta Urquiaga (2019), sucede en el Restaurant Don Cuy, puesto que, en la evaluación de los costos éstos se desconocen, es decir, no se tiene conocimiento sobre el desglose de cómo están distribuidos los costos, es por ello, que según estas y otras fallas encontradas se propone el diseño e implementación del sistema de costeo para el referido restaurant.

De acuerdo a lo encontrado, es necesario que en todo negocio y en este caso específico del rubro del restaurant, que los dueños y colaboradores tengan conocimientos; puesto que esto redundará en el sentido común de compra de materiales e insumos, gastos, utilidades, logística del negocio, asuntos administrativos y contables, entre otros aspectos.

Olivera (2019), quien en su estudio en el Restaurant Turístico Tradiciones SAC, de Chiclayo; encontraron como hallazgo que la entidad ejecuta un sistema de costos tradicional; lo cual genera problemas en el manejo de costos; en ese sentido, no realiza una adecuada determinación de los costos. Por otra parte, se aplicó un sistema de costo, repercutiendo de forma significativa a la distribución que se realizan durante el procesamiento de costo del referido restaurante.

Similar a los problemas que presenta Olivera (2019), sucede en el Restaurant Don Cuy en el que se visualizó que el costo lo realizan de manera empírica y que no llevan un control en la distribución de éste en el costo de los platos, los insumos, gastos básicos, entre otros; de igual manera, se propuso el diseño e implementación del sistema de costeo por órdenes que contribuya a llevar un mejor control e incida en su buena gestión económica.

Adicionalmente, a la falta de conocimientos y distribución de los costos de producción, en la descripción de los costos se encontró, que dichos costos no permiten establecer cuál es el precio de venta de cada plato, la ganancia y la utilidad; por lo que no se estiman como costos de producción; requiriendo de este modo un sistema de costeo para llevar un mejor control.

Fonseca (2018), quien, en su estudio realizado en el Restaurante Paradero Don Gabriel en Ecuador, obtuvo que al tener los análisis de costos por platos y bien precisados sus precios, estos se vuelven más competitivo, ya que se basa en valores más reales" (Fonseca, 2018, p. 66).

De acuerdo con lo anterior, es importante observar que el análisis de costo ejecutado por Fonseca (2018), fue de gran aporte debido a que se tiene en consideración los costos de los platos con sus precios desglosados con costos de materia prima, costos indirectos, etc., lo que incide es que éstos sean más reales.

Es de relevancia lo desarrollado por Fonseca (2018), para esta investigación puesto que marca un precedente acerca de que teniendo los precios bien fijados por platos el valor de éste es más real y resulta competitivo para otras organizaciones del mismo rubro. 
Perata, Freitas y Pesce (2016), en su investigación fijaron como propósito identificar y cuantificar los costos de cada uno de los productos vendidos y mejorar la manera en la toma de decisiones en cuanto al precio de venta de los productos; estos autores desarrollaron un método para fijar los precios de ventas por cada producto basado fundamentalmente en sus costos.

En ese mismo orden de ideas, lo recomendado por los autores citados anteriormente constituye un aporte fundamental, debido a que, al tener un buen control y fijación de costos en el Restaurant Don Cuy, se puede decidir respecto a los precios, asimismo, a la toma de decisiones.

Cárdenas y Rodríguez (2018), quienes, en su investigación sobre el Restaurant del Sanatorio de Agua de Dios, ubicado en Colombia, expresaron que: "la entidad maneja un sistema de costos que centra su operación en el cálculo de los costos por unidad funcional en la que se puede identificar un costeo general de los elementos indirectos y mano de obra" (p. 17).

Lo anteriormente descrito, es lo que se estimó realizar cuando se hizo la descripción de los costos en el Restaurant Don Cuy; no obstante, los costos obtenidos no reflejan los costos reales de producción, es por ello, que se consideró diseñar el sistema de costeo por órdenes, para tener un mejor control.

Olivera (2019), quienes, en el Restaurant Turístico Tradiciones S.A.C. de Chiclayo, estimaron los costos relacionados a la preparación de sus platillos; por lo que "establecieron un valor para su venta que fuera el más adecuado, tanto para la empresa como para sus comensales; todo lo cual se vea reflejado en el beneficio del cliente" (p. 7).

Lo expresado por Olivera (2019), es de gran aporte para esta investigación, puesto que, al tener los precios justos y adecuados de los potajes, los clientes y la empresa serán beneficiados.

Asimismo, Facundo (2018), sobre la problemática del restaurant turístico El Sabor de Mi Tierra, ubicado en San Ignacio en Cajamarca; estableció que este, en su momento no tenía un sistema de costeo apropiado; en ese sentido, el modo para estimarlos era de forma empírica; es por ello que no se tenía un valor fijo de lo correspondiente a los ingresos por plato de comida. (p. 17)

Similarmente a lo que se encontró en el Restaurant Don Cuy, Facundo (2018), en su investigación encontró que empleaban métodos empíricos para estimar los costos.

Es importante el considerar los costos fijos, puesto que se tiene la observación de la rentabilidad y las ganancias que genera el negocio; es por ello, que se decidió diseñar el sistema de costeo por órdenes en el Restaurant Don Cuy, lo cual impactará de manera positiva en el negocio.

Con relación a diseñar una propuesta de costeo por órdenes de producción que le permita llevar un control de los principales platos de comida del Restaurant "Don Cuy", Huaraz - 2021. a través de los instrumentos empleados y la guía de entrevista, permitieron realizar un diagnóstico inicial, que contribuyó a verificar las falencias que se presentan en el Restaurant Don Cuy; debido a las debilidades presentadas se consideró la propuesta de un sistema de costeo por órdenes que le permita tener valores reales de los platos, de la materia prima, mano de obra y de servicios involucrados en lo que abarca a los costos del restaurant y que éstos a su vez redunden en la utilidad del negocio, la toma de decisiones, asimismo, mejore la competitividad frente a la competencia basados fundamentalmente en el manejo de valores reales

Fonseca (2018), en su estudio realizado en el Restaurant Paradero Don Gabriel, ubicado en Ecuador; al contar con un diseño de herramienta de costos que ayude a la asignación de precios, no fue necesario generar un método de costeo; sin embargo, éste demostró que al tener dicho sistema "la asignación de precios se vuelve más competitivo ante la competencia, ya que se basa en valores más reales" (Fonseca, 2018, p. 66).

A diferencia del estudio de Fonseca (2018), en la que no se elaboró un sistema de costeo porque ya contaban con uno, en el caso de este estudio, en el Restaurant Don Cuy, si debió realizarse el sistema de costeo por órdenes para llevar un mejor control de los costos.

Urquiaga (2019), quien en su estudio en el Restaurant El Dorado ubicado en Chimbote, cuyo propósito fue conocer cómo es que se vienen asignando los costos de producción en el Restaurant El Dorado, así mismo conocer cuál es su margen de ganancia por cada plato que ofrece” (p.11); es por ello, que elaboró un sistema de costeo por órdenes de producción, el cual le "permitió reconocer 
su utilidad y sus ganancias; dicho sistema favoreció en la estimación de la materia prima, mano de obra y costos indirectos, además de conocer la ganancia por cada plato" (p. 32).

El problema y hallazgo de Uquiaga (2019), son relevantes para esta investigación, puesto que con el sistema de costeo propuesto en el Restaurant Don Cuy se pudo establecer el mejor control de los costos en el referido restaurant y precisar el cálculo de los costos de materia prima, costos indirectos y de servicios.

Perata, Freitas y Pesce (2016), quienes desarrollaron un método para la fijación de precios de ventas para una empresa multiproducto de gastronomía, fundamentado principalmente en los costos. Por lo que la empresa realizó el análisis para establecer una estructura de costos; en la que se propuso un modelo para mejorar en base a las necesidades detectadas en el diagnóstico.

De manera similar a lo efectuado por Perata, Freitas y Pesce (2016), en el Restaurant Don Cuy se propuso un sistema de costeo por órdenes que permitió tener un mejor seguimiento y control a la distribución del costo. Esto es de gran relevancia, puesto que, contribuye a llevar un mejor orden en los precios, ganancias y utilidades de la organización.

\section{CONCLUSIONES}

Una vez culminado el proceso investigativo, las conclusiones que se han derivado del presente estudio son las siguientes:

- Se describieron los elementos del costo de producción de los platos más vendidos mediante el costeo empírico, donde se concluye que el restaurant no contaba con un sistema de costeo adecuado a su rubro; además de no considerar todos los costos reales en los que refleja la empresa al no identificarlos, asimismo los trabajadores no se encontraban en planilla por ende no estaban pagándoles sus beneficios laborales, ya para finalizar se observó el manejo de un margen de costo del $12 \%$ para el chancho al palo, del $11 \%$ para el chancho al cilindro y el $14 \%$ para el don cuisano ya que son los platos más consumidos por el cliente.

- Se analizaron los costos manejados por el restaurant, en el que se presentan debilidades el cual se observó a través de los instrumentos aplicados los errores al no tener conocimientos sobre cuáles son sus costos reales entre las que destacan: no poseen una metodología de costos para recepcionar las materias primas, asimismo, no realizan el seguimiento para la cuantificación de los platos vendidos, también se observó que no se consideraron los materiales auxiliares y el costo de taxi al momento de realizar las compra. Por otra parte, se vio la diferencia de $\mathrm{S} / 2.00$ según costeo empírico y propuesta, obteniendo una ganancia de S/13.00 para el chancho al palo, S/12.00 para el chancho al cilindro y S/6.00 para el don cuisano; donde se observa que para la elaboración del plato le está costando la mitad de precio de carta y por último los desperdicios no se consideraron como un ingreso para recuperar parte del costo; siendo necesaria la implementación de un sistema de costeo para tener un mejor costeo.

- Debido a que la empresa no cuenta con un sistema de costo, se propuso un sistema de costeo por órdenes que permitió tener valores reales de los platos, de la materia prima, mano de obra y de servicios involucradas en lo que abarca a los costos del restaurant y que éstos a su vez se reflejen en la utilidad del negocio, la toma de decisiones, asimismo, mejore la competitividad basados en fundamentos del manejo de valores reales. 


\section{RECOMENDACIONES}

Posteriormente a las conclusiones obtenidas en este estudio, se sugieren las recomendaciones siguientes:

- Se recomienda al gerente del Restaurant Don Cuy, reconocer el costo de producción de cada orden, asimismo, el costo de la materia prima, mano de obra y de los costos indirectos de fabricación para la elaboración de cada plato.

- Se recomienda tener en cuenta los elementos del costo para determinar el precio real de cada plato que se ofrece, por ende, realizar un análisis para un adecuado control y ver la capacidad de producción para que pueda obtener una mayor utilidad.

- Se recomienda al gerente del Restaurant Don Cuy, ejecutar el sistema de costeo por órdenes propuesto por órdenes específicas, el cual permitirá conservar un control apropiado del costo, redundando en la toma de decisiones para el precio real de sus platos.

- Se sugiere al gerente del Restaurant Don Cuy, que para evitar sanciones y multas por la entidad competente en el cumplimiento de las normativas de desperdicios establecidos en la Ley $\mathrm{N}^{\circ} 1278$ sobre Gestión Integral de Residuos Sólidos, ya que cuentan con desperdicios que son vendidos como comida para cerdos.

\section{REFERENCIAS}

Afifah, N., \& Gunawan, A. (2020). Perhitungan Harga Pokok Produksi Menggunakan Metode Costos de órdenes de trabajo (Studi Kasus Pada Usaha Konveksi "Mowin Concept”). Indonesian Accounting Literacy Journal, 1(1), 139-150. Obtenido de https://jurnal-akuntansi.polban.ac.id/ialj/article/view/181/59

Alfarisi, S., \& Boediono, G. (2021). Evaluasi Penerapan Job Order Costing dalam Penentuan Harga Pokok Produksi. Sm all Business Accounting Management and Entrepreneurship Review, 1(1), 46-55. Obtenido de http://dhsjournal.id/index.php/SBAMER/article/view/50

Almeida, A., \& Cunha, J. (2017). The implementation of an Activity-Based Costing (ABC) system in a manufacturing company. Procedia Manufacturing, 13, 932-939. Obtenido de https://www.sciencedirect.com/science/article/pii/S2351978917307990

Amancio, M., \& Keller, A. (2018). Analysis of the percentage cost of the merchandise sold from the preparations of a self service restaurant. Lecturas: Educación física y deportes, 23(246), 72-84. Obtenido de https://dialnet.unirioja.es/servlet/articulo?codigo $=7272832$

Ancash Noticias. (20 de junio de 2020). «Don Cuy» atiende por delivery con protocolos de seguridad y estrena aplicativo para pedidos. Obtenido de https://bit.ly/2G3I8Ub

Arellano, Otto, Quispe, Gabith, Ayaviri, Dante, Escobar, Fortunato. (2017). Study of the Application of The ABC Cost Method in the Mypes of Ecuador. Revista de Investigaciones Altoandinas, 19(1), 33-46. http://www.scielo.org.pe/scielo.php?script=sci_arttext\&pid=S2313-29572017000100004

Arias, L.; Portilla, L., Bernal, M. (2008). Los costos y su manejo con el control estadístico de procesos, con ayuda de la distribución normal. Scientia et Technica, Año XIV, No 38, 259-263. https://www.redalyc.org/pdf/849/84903845.pdf

Arias, F. (2006). El proyecto de investigación. Introducción a la metodología científica. Séptima Edición. Caracas, Venezuela: Editorial Episteme.

Artieda, C. (2015). Análisis de los sistemas de costos como herramientas estratégicas de gestión en las pequeñas y medianas empresas (PYMES). Revista Publicando, 2(2), 90-113. https://revistapublicando.org/revista/index.php/crv/article/view/31

Balestrini, M. (2006). Como se elabora el proyecto de investigación. Caracas, Venezuela: BL Consultores y Asociados.

Bunea, C. (2013). He cost of production under direct costing and absorption costing - a comparative approach. Revista „ACADEMICA BRANCUSSI” PUBLISHER, 123-129. Obtenido de https://www.utgjiu.ro/revista/ec/pdf/201302/19_Bunea\%20Bontas\%20Cristina.pdf

Cardénas, L., \& Rodríguez, D. (2018). Sistema de costos ABC para el servicio de Restaurante del Sanatorio de Agua de Dios. Repositorio de Corporación Universitaria Minuto de Dios. Tesis de Especialista en Proyectos. Girardpt, Colombia. Obtenido de https://bit.ly/34fyvK3

Cárdenas, R. (2016). Costos 1. Ciudad de México: Instituto Méxicano de Contadores Públicos.

Casanova, E. (2020). Gestión de costos y la eficiencia en las ventas del servicio de restaurante en la empresa de Chuck E Cheese's, Trujillo 2019. Repositorio Digital Universidad César Vallejo. Trujillo, Perú. Tesis de Maestría en Administración de Negocios. Obtenido de https://bit.ly/36lWCJt 
Capa, Lenny, García, María, Herrera, Alexander. (2019). Consideraciones a los tipos de costeo de la producción para la responsabilidad social empresarial. Revista Universidad y Sociedad, 11(5), 368-372. Recuperado de http://scielo.sld.cu/scielo.php?script=sci_arttext\&pid=S2218-36202019000500368\&lng=es\&tlng=es.

Chacón, Galia (2016). Costeo por operaciones: Aplicación para la determinación de precios justos. Actualidad Contable FACES, Año 19 No 32, 5-39. https://www.redalyc.org/pdf/257/25744733002.pdf

Crespo, E., Chica, G., Astudillo, C., Patiño, A., \& Tapia, J. (2020). Application of an ABC Costing Model to an MSME Company in the Manufacturing Sector into the UDA ERP Administrative System. Advances in Intelligent Systems and Computing, 1216, 341-347. Obtenido de https://doi.org/10.1007/978-3-030-51981-0_43

Dal, C., Manfroi, L., Bortoli, L. \& Theisen, C. (marzo, 2019). Formação do preço de venda na indústria de panificação. Costos y Gestión (96). Recuperado de http://www.iapuco.org.ar/revista-costos-y-gestion/2019-04-A29Nro96.pdf

Dewi, K., Musmini, L., \& Marvilianti, P. (2019). Analisis perbandigan penggunaan job order costing method dan process costing method untuk meningkatan akurasi laba usaha. Jurnal Ilmiah Mahasiswa Akuntansi, 1(10), 6171. Obtenido de https://ejournal.undiksha.ac.id/index.php/S1ak/article/view/20537

Dewi, Y., Tripaupi, L. E., \& Zukhri, A. (2017). Analisis metode job order costing dalam menentukan harga pokok produksi pada srada Lamp's di Gianyar. Jurnal Pendidikan Ekonomi Undiksha, 9(2), 516-525. Obtenido de https://ejournal.undiksha.ac.id/index.php/JJPE/article/view/20146/12160

Dumitru, E., \& Ene, M. (2018). The Importance of the ABC Costing Method for the Strategic Analysis of Public Passenger Transport. Social Economic Debates, 7(2), 43-47. Obtenido de http://www.economicdebates.ro/art\%207\%20Dumitru\%20DSE\%202\%202018.pdf

Facundo, J. (2018). Implementación de un sistema de costos para incrementar la rentabilidad del restaurant turístico El Sabor de Mi Tierra, San Ignacio - 2017. Repositorio Digital Universidad César Vallejo. Chiclayo, Perú. Tesis de Contaduría Pública. Obtenido de https://bit.ly/2SeWOlQ

Falconí, M. (2014). La importancia de los sistemas de costos tradicionales y modernos para fijar precios de venta al público y conseguir el desarrollo empresarial. Journal Espe, 1(1), 63-64. Obtenido de https://journal.espe.edu.ec/ojs/index.php/Sigma/article/view/952/566

Fonseca, W. (2018). Evaluación de los costos y su influencia en la fijación de precios de las comidas y bebidas en el Restaurant Paradero Don Gabriel. Repositorio de la Universidad Pontificia de Ecuador. Ambato, Ecuador. Tesis de Ingeniería en Contabilidad y Auditoría. Obtenido de https://bit.ly/2HBUAuu

Gersil, A., \& Kayal, C. (2016). A comparative analysis of normal costing method with full costing and variable costing in internal reporting. Revista International Journal of Management (IJM), 7(3), 79-92. Obtenido de https://iaeme.com/MasterAdmin/Journal_uploads/IJM/VOLUME_7_ISSUE_3/IJM_07_03_009.pdf

González, N. (2017). Procedimiento de un sistema de costo. Cofín Habana, 12(2), 91-101. Obtenido de http://scielo.sld.cu/pdf/cofin/v11n2/cofin07217.pdf

Gotuzzo, R. (2018). Metodología de la investigación: elaboración de diseños para contrastar hipótesis. Lima: San Marcos.

Greenberg, R., \& Schneider, A. (2019). Job order costing: a simulation and vehicle for conceotual discussion. Academy of Educational Leadership Journal, 14(3), 39-48. Obtenido de https://www.proquest.com/openview/6599b24a16b1c2236baf6b8f4b5e65a8/1?pqorigsite $=$ gscholar $\&$ amp; $\mathrm{cbl}=38741$

Hasan, S. (2015). Variable Costing and Its Applications in Manufacturing. Revista International Scholar Journal of Accounting and Finance, 1(1), 1-10.

Hilmiyati, F., Zahara, M., Mulyani, S., \& Suriyanti, L. (2020). Peranan job order costing dalam menentukan hpp dan harga jual studi kasus pada Percetakan Berkah. Halaman, 4(1), 561-571. Obtenido de https://jptam.org/index.php/jptam/article/view/500/445

Idris DM, M. (2019). Diseño de sistemas, costos de orden de trabajo. Arca de la innovación, 2(2), 53-65. Obtenido de https://ojs.umrah.ac.id/index.php/bahterainovasi/article/view/1627/771

Innocent, N., \& Chimezie, N. (2013). Principles of costing and cost analysis as a tool for production costs control: a case study of Nigerian companies. Revista Research Journal in Engineering and Applied Sciences, 2(3), 225229. Obtenido de https://www.researchgate.net/profile/Innocent-

Nnanna/publication/330486228_PRINCIPLES_OF_COSTING_AND_COST_ANALYSIS_AS_A_TOOL_FO R_PRODUCTION_COSTS_CONTROL_A_CASE_STUDY_OF_NIGERIAN__COMPANIES/links/5c424436 458515a4c7308bdc/PRINCIPLES-OF-COSTING-AND-COST-ANALYSIS-AS-A-TOOL-FORPRODUCTION-COSTS-CONTROL-A-CASE-STUDY-OF-NIGERIAN-COMPANIES.pdf

Kato, L. (2013). The problem of the profit in economic theory and business accounting. Revista Economy Informs, 2013(283), 23-63.

Latorre, F. (2016). Estado del Arte de la Contabilidad de Costos. Revista Publicando, 3(8), 513-528. https://revistapublicando.org/revista/index.php/crv/article/view/317

Ley de Gestión Integral de Residuos Sólidos $N^{\circ}$ 1278. (2017). https://busquedas.elperuano.pe/normaslegales/apruebanreglamento-del-decreto-legislativo-n-1278-decreto-decreto-supremo-n-014-2017-minam-1599663-10/

Leite, A. \& Renauld, B. (mayo, 2019). Production cost of autologous platelet rich plasma gel. Revista LatinoAmericana Enfermagem (27). Recuperado de https://www.scielo.br/pdf/rlae/v27/0104-1169-rlae-27-e3221.pdf 
Lima, F., \& Araújo, R. (2016). Gestão estratégica de custos: custeio por absorção em pequenas empresas em Recife, PE, Brasil. Revista Interações (Campo Grande), 17(3), 528-541. Obtenido de https://www.scielo.br/scielo.php?pid=S1518-70122016000300528\&script=sci_abstract\&tlng=pt

Macias, J., Reyes, M., \& Hurel, G. (2020). Absorption costs and their effect on the results of the company DIMETAL S.A. Observatory Magazine of the Latin American Economy, 1-14. Obtenido de https://www.eumed.net/rev/oel/2019/06/costos-absorcion-dimetalsa.html

Mejía, E., Guzmán, N., \& Hernández, R. (2016). Costos de producción de servicios en los restaurantes de la ciudad de San Lorenzo Departamento de Boaco, en el II semestre del año 2015. Repositorio Institucional UNANManagua. Tesis de Contaduría Pública y Finanzas. Juigalpa, Managua. Obtenido de https://bit.ly/2GkG4a6

Ministerio de Economía y Fnanzas-MEF. (2016). Métodos de Costeo: Costeo Absorbente vs Costeo Variable. Obtenido de https://bit.ly/312cgOA

Morillo, M. (2002). Diseño de Sistemas de Costeo: Fundamentos Teóricos. Revista Actualidad Contable FACES, 5(5), 7-22. Obtenido de https://www.redalyc.org/pdf/257/25700507.pdf

Morrillo, M., \& Cardozo, C. (2016). Sistema de costos basado en actividades en hoteles cuatro estrellas del estado Mérida, Venezuela. INNOVAR. Revista de Ciencias Administrativas y Sociales, 27(64), 91-113. Obtenido de https://www.redalyc.org/jatsRepo/818/81850404010/index.html

Mulyanda, M., \& Evayani, E. (2019). Perancangan aplikasi perhitungan harga pokok produksi dengan metode job order costing pada Meudang Perkasa Furniture. Jurnal Ilmiah Mahasiswa Ekonomi Akuntansi, 4(3), 428-440. Obtenido de http://www.jim.unsyiah.ac.id/EKA/article/view/12576/pdf

Namazi, M. (2016). Time-driven activity-based costing: Theory, applications. Revista Iranian Journal of Management Studies (IJMS), 9(3), 457 - 482. Obtenido de https://ijms.ut.ac.ir/article_57481_793361023886e0c6698746124227687b.pdf

Nima, J. (2018). Propuesta de un proceso de costos dentro de un modelo de gestión para mype del subsector restaurantes en Lima Metropolitana. Repositorio Universidad de Peruana de Ciencias Aplicadas. Lima, Perú. Tesis de Ingeniería Industrial. Obtenido de https://repositorio.ucv.edu.pe/bitstream/handle/20.500.12692/37251/AC_Novoa_VSE-Guti\%C3\%A9rrez_PEBerm\%C3\%BAdez_GOV.pdf?sequence=1\&amp;isAllowed $=y$

Novoa, S., Gutiérrez, E., \& Bermúdez, O. (2017). Propuesta de un sistema de costos por órdenes específicas para mejorar la rentabilidad en conservas de caballa en la empresa Oldim S.A. - Chimbote 2016. Revista Ingeniería: Ciencia, Tecnología e Innovación, 1-16. Obtenido de https://bit.ly/3jlriyu

Olivera, L. (2019). Sistema de costeo ABC para la determinación de los costos del Restaurant Turístico Tradiciones SAC. Chiclayo 2018. Repositorio Universidad César Vallejo. Tesis de Contaduría Pública. Chiclayo, Perú. Obtenido de https://bit.ly/347h7qM

Parra, J., Mondragón, S., \& Peña, Y. (2014). Costeo variable vs. Costeo por absorción. Retomando una vieja forma de su enseñanza. Revista Activos (22), 111-136. https://revistas.usantotomas.edu.co/index.php/activos/article/view/2790

Peralta, D. (2016). Aplicación de un sistema de costos para mejorar la rentabilidad del Restaurant J\&L S.A.C., Bagua 2015. Repositorio Institucional Universidad Señor de Sipán. Pimentel, Perú. Tesis de Contaduría Pública. Obtenido de https://bit.ly/3cWcbct

Perata, A., Freitas, H., \& Pesce, G. (2016). Cost-Based Information System for Price Decision-Making: A case study of a multi-processing company of the gastronomic sector. Escritos Contables y de Administración, 7(2), 13-69. Obtenido de https://revistas.uns.edu.ar/eca/article/view/532

Polimeri, R., Fabozzi, F., Adelberg, A., \& Kole, M. (1997). Contabilidad de costos. Tercera edición. Colombia: McGraw-Hill.

Putri, F., \& Desi, E. (2019). Cost of production price analysis using job order costing method in CV. Alam Lestari Jaya. International Journal of Information System \& Technology, 4(1), 349-353. Obtenido de http://ijistech.org/ijistech/index.php/ijistech/article/view/70/70

Quiñonez, E. (2020). Scientific and philosophical approach to the application of an ABC costing model as an alternative for the development of the Ecuadorian agricultural sector. Revista INNOVA Research Journal, 5(2), 174-185. Obtenido de https://dialnet.unirioja.es/servlet/articulo?codigo $=7475502$

Reveles, R. (2019). Análisis de los elementos del costo. Segunda edición. Ciudad de México: Instituto Mexicano de Contadores Públicos.

Rincón, C. (2011). Costos para Pyme. Bogotá, Colombia: Eco Ediciones.

Rincón, C., Sánchez, X., \& Cardona, L. (2019). Clasificación teórica de los costos. Revista Escuela de Administración de Negocios, 87(1), 193-206. Obtenido de http://www.scielo.org.co/pdf/ean/n87/0120-8160-ean-87-193.pdf

Rincón, H. (2005). Contabilidad de costos y de gestión en la industria farmacéutica venezolana: Estudio de un caso. Revista Venezolana de Gerencia, 10(30), 267-287. Obtenido de https://www.redalyc.org/pdf/290/29003006.pdf

Rojas, R. (2007). Sistemas de Costos un proceso para su implementación. Manizales: Universidad Nacional de Colombia Sede Manizales.

Samón, J., Cautín, L., Mustelier, L., \& Caraballo, L. (2018). Necesidad de estudios sobre costos en la Universidad de Ciencias Médicas Guantánamo. Revista Información Científica, 97(1), 76-85. Obtenido de http://scielo.sld.cu/scielo.php?script=sci_abstract\&pid=S1028-99332018000100076\&lng=es\&nrm=iso 
Sitanggang, D., Sion, N., \& Suryanti, L. (2020). Penerapan Metode Job Order Costing Dalam Penentuan Harga Jual Produk Pada UMKM Gemilang Jaya. Jurnal Akuntansi dan Ekonomika, 10(2), 168-179. Obtenido de https://ejurnal.umri.ac.id/index.php/jae/article/view/1960/1350

Torres, Carlos, Salete, María y Delgado, Constanza. (2017). Costeo de productos en la industria panadera utilizando el método ABC. Interciencia, 42 (10): 646-652. Disponible en: https://www.redalyc.org/articulo.oa?id=33953313004

Tran, T., \& Thao, N. (2020). Factors affecting the application of ABC costing method in manufacturing firms in Vietnam. Management Science Letters, 10, 2625-2634. Obtenido de http://growingscience.com/beta/ms1/3844factors-affecting-the-application-of-abc-costing-method-in-manufacturing-firms-in-vietnam.html

Urquiaga, N. (2019). Costos de producción para determinar el margen de ganancia del restaurante "El Dorado" - Nuevo Chimbote Periodo 2019. Repostorio Digital Universidad César Vallejo. Tesis de Contaduría Pública. Chimbote, Perú. Obtenido de https://bit.ly/3cIKpQg

Valderrama, S., \& Jaimes, C. (2019). El desarrollo de la tesis: decriptiva- comparativa, correlacional y cuasiexperimental . Lima: San Marcos.

Yardin, A. (2002). A review of the general theory of cost. Revista Contabilidade y Finanças - USP, $30(1), 71$ - 80. Obtenido de https://www.scielo.br/pdf/rcf/v13n30/v13n30a06.pdf 